\title{
Compact Personal Distributed Wearable Exposimeter
}

\author{
Peter Vanveerdeghem, Patrick Van Torre, Arno Thielens, Jos Knockaert, Member, IEEE, \\ Wout Joseph, Senior Member, IEEE, Hendrik Rogier, Senior Member, IEEE
}

\begin{abstract}
A compact wearable Personal Distributed Exposimeter is proposed, sensing the power density of incident radio-frequency ( $R F)$ fields on the body of a human. In contrast to current commercial exposimeters, our Personal Distributed Exposimeter, being composed of multiple compact personal wearable RF exposimeter sensor modules, minimizes uncertainties caused by the proximity of the body, the specific antenna used and the exact position of the exposimeter. For unobtrusive deployment inside a jacket, each individual exposimeter sensor module is specifically implemented on the feedplane of a textile patch antenna. The new wearable sensor module's high-resolution logarithmic detector logs RF signal levels. Next, on-board flash memory records minimum, maximum and average exposure data over a time span of more than two weeks, at a one-second sample period. Sample-level synchronization of each individual exposimeter sensor module enables combining of measurements collected by different nodes. The system is first calibrated in an anechoic chamber, and then compared to a commercially available single-unit exposimeter. Next, the Personal Distributed Exposimeter is validated in realistic conditions, by measuring the average RF power density on a human during a walk in an urban environment and comparing the results to spectrum analyzer measurements with a calibrated antenna.
\end{abstract}

Index Terms - dosimetry, radio frequency exposure, textile antennas, wearable electronics

\section{INTRODUCTION}

$\mathrm{N}$ ational legislation and the international Commission on Non-Ionizing Radiation Protection (ICNIRP) [1] impose limits in terms of whole-body averaged SAR (SARwb) [2]. Since these SARwb levels can only be evaluated by numerical simulations [3], equivalent reference levels, which can be measured and compared to the international guidelines issued by INCIRP, have been defined on the incident power density. Such exposure measurements are currently performed with commercially available Personal ExposiMeters (PEMs) [2],[4]-[7]. These measurements are compromised by large

This paper is submitted for review on the $15^{\text {th }}$ December 2014. This research was partially funded by the Inter-University Attraction Poles Program initiated by the Belgian Science Policy Office.

P. Vanveerdeghem, P. Van Torre, A. Thielens, W. Joseph, and H. Rogier are with the Department of Information Technology, Ghent University/iMinds, Ghent, B-9000, Belgium (e-mail: peter.vanveerdeghem@ugent.be)
P. Vanveerdeghem, and J. Knockaert are with the Department of Industrial System and Product Design, Ghent University, Kortrijk, B-8500, Belgium

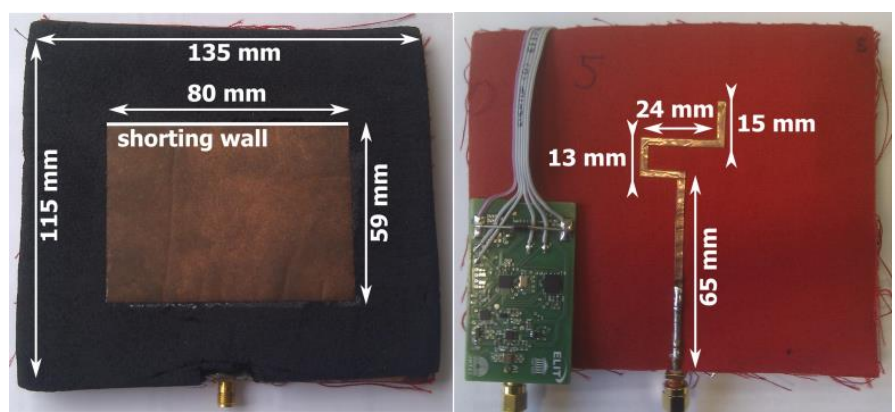

Fig. 1. Top view (left) and bottom view (right) of the patch antenna. On the bottom view, the active circuit and antenna feed are shown separately. In the actual implementation, the exposimeter's electronics are mounted onto the feed plane of the antenna.

measurement uncertainties, due to shadowing by the test person's body [8], [9], dependence on polarization [10] and out-of-band detection. Furthermore, conventional PEMs cannot be unobtrusively deployed on the human body, whereas a fully integrated wearable on-body PEM enables continuous monitoring of long-term RF exposure, without hindering the test person's daily activities. Moreover, an onbody Personal Distributed Exposimeter (PDE), composed of multiple PEMs, increases the measurement accuracy [11].

We present a fully autonomous on-body PDE, composed of multiple independent RF-exposure modules, each integrated on a textile antenna feed plane. Thanks to their ground plane [12], the applied patch antennas minimize capacitive antenna loading by the body. Without loss of generality, we specifically configure the exposimeter for the Global System for Mobile Communications (GSM) worldwide down-link [925 MHz-960 MHz] frequency band [13].

Each wearable exposimeter module makes use of a state-ofthe-art logarithmic-detector to pair accuracy with a dynamic range of $80 \mathrm{~dB}$. The on-board flash memory logs for over two weeks of measurement data, thereby eliminating a permanent Personal Computer (PC) connection. A similar on-body device was documented in [11], yet without data logging or unobtrusive integration potential. To the authors' knowledge, this is the first fully tested wearable PDE.

In Section II, the wearable PDE system is described, followed by its validation on a human body in Section III. The calibration procedure is explained in Section IV. A real life measurement is outlined in Section V. Conclusions are listed in Section VI. 


\section{SYSTEM OVERVIEW}

The PDE is composed of multiple newly designed wearable exposimeter modules, of which the construction is described below.

\section{A. Antenna}

Due to the large wavelength of the frequency of the GSM downlink band, an aperture-coupled shorted patch antenna is selected, as displayed in Fig. 1. The antenna features a compact size and excellent antenna performance in proximity of the human body, while avoiding fragile probe feed connections [14], [15]. Therefore, this antenna is more suitable for garment integration than conventional antennas. Further improvement of the coupling between the antenna and the exposimeter module is obtained by employing an $\mathrm{H}$-shaped coupling slot, thereby also minimizing the backward radiation into the human body [16]. The textile material is flexible and lightweight, without sacrificing antenna performance, in comparison to rigid antennas. A flexible polyurethane protective foam (thickness $=11 \mathrm{~mm}$ permittivity $\varepsilon_{\mathrm{r}}=1.16$ and $\tan \delta=0.010$ ), commonly used in protective garments for rescue workers is applied as a substrate material, protecting the electronic circuitry from external factors, such as heat and humidity. A low-cost, conductive, electro-textile material, called Flectron, is used to construct the ground plane and radiating element. The material has a thickness less than 0.25 $\mathrm{mm}$ and surface resistivity less than $0.1 \Omega / \mathrm{sq}$., minimizing conductor losses. The influence of the body, which is in close proximity to the antenna, is limited, thanks to the ground plane structure. This feature makes the PDE's performance nearly independent from the test person's body morphology. Measurements performed on another person produced similar results [17],[18]. The feed substrate is constructed using two stacked Aramid textile layers, a material which is frequently used as outer layer in firefighter jackets (thickness $=0.95 \mathrm{~mm}$, permittivity $\varepsilon_{\mathrm{r}}=1.97$ and $\tan \delta=0.02$ ). The feed line is realized by means of copper foil. The antenna covers the complete GSM $935 \mathrm{MHz}$ to $960 \mathrm{MHz}$ downlink band. The 3 $\mathrm{dB}$ beam width of the antenna approximately equals $110^{\circ}$, with an antenna gain of $2.9 \mathrm{dBi}$ and an antenna efficiency of $76.6 \%$. The top view of the antenna and its dimensions are shown in Fig. 1, with the electronic circuitry integrated onto the feed plane of the antenna. A detailed construction diagram of the antenna is shown in Fig. 2.

By placing the electronics on the feed plane of the patch antenna, each very compact module may be unobtrusively integrated into garments or clothing [19]. In addition, each unit can be encapsulated together with the textile antenna into a breathable Thermoplastic PolyUrethane coating (TPU), implementing a washable system [20].

\section{B. System design}

The block diagram of the proposed exposimeter module, integrated on the antenna feed-plane, is presented in Fig. 3. The selection of the key components is motivated as follows.

The RF-signal received by the antenna is filtered by a Surface Acoustic Wave (SAW) Bandpass filter (BPF), to

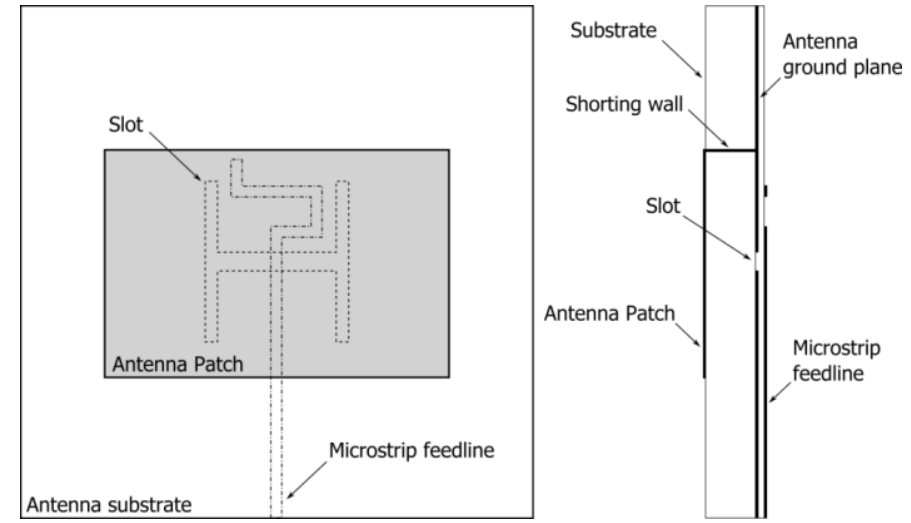

Fig. 2. Top view (left) and cross-section (right) of the patch antenna.

remove undesired out-of-band signals. In this configuration for the GSM down-link, the TriQuint 856528 SAW filter is chosen, with a bandwidth of $35 \mathrm{MHz}$ and passband insertion loss of $2.5 \mathrm{~dB}$. Outside the passband, an attenuation of $35 \mathrm{~dB}$ is quickly achieved. Thanks to the filter architecture, no additional impedance matching network is required, helping to reduce the physical size of the circuit. The filtered signal is measured by an Analog Devices ADL5513 [21] Logarithmic RF Detector, providing an output voltage proportional to the input $\mathrm{RF}$ level in $\mathrm{dBm}$ over a very large dynamic range. The output voltage of the RF detector is measured by a 16 bit

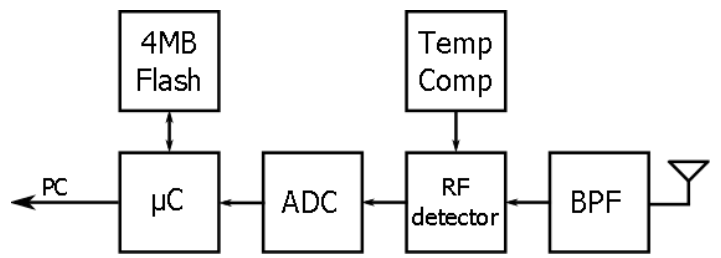

Fig. 3. Bock diagram of single autonomous exposimeter module, with integrated band-pass filter (BPF), logarithmic RF detector and temperature compensation (Temp Comp). The PC connection is only used to configure the microcontroller $(\mu \mathrm{C})$ and to download logged data from the $4 \mathrm{MB}$ onboard flash memory.

Analog-to-Digital Converter (ADC) and transported on the PDE board to the C8051F921 microcontroller $(\mu \mathrm{C})$, by Silicon Labs, through the high speed Serial Peripheral Interface (SPI) data bus. To correct the small temperature-dependent variation of the output level of the Logarithmic RF Detector, an automatic frequency-dependent temperature compensation is implemented by a Digital-to-analog-converter (DAC) connected to the on-board microcontroller and its built-in temperature sensor. The micro controller is programmed in C. The software is uploaded to its memory through the InCircuit-Programming interface.

The digitized measurement data are further processed by the on-board software at a data rate of $1000 \mathrm{samples} / \mathrm{sec}$. The minimum, maximum, as well as arithmetic- and geometric averages of the received RF signal power, over a one-second time slot, are stored into the on-board flash memory. The 4 MB flash memory provides up to two weeks of non-volatile storage space. To guarantee the data integrity, at each time slot, a check sum of the measurement data is calculated and stored into the memory together with the measured data.

Besides the storage of the averaged measurement data at a 


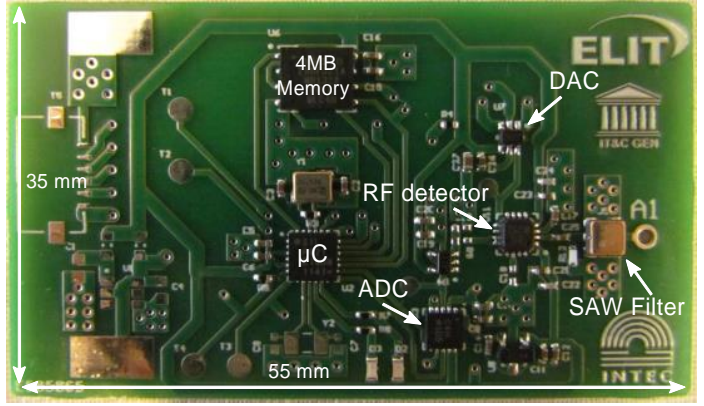

Fig. 4. Top view of the system $[57 \mathrm{~mm} \times 33 \mathrm{~mm}]$

one-second rate, the system may store raw sampled data at a full sample rate of 1000 samples per second for analysis of high-speed measurements in post processing. The maximum sample rate of the exposimeter is $250 \mathrm{ksps}$, as determined by the ADC specifications. After the logging period, the measurement data are easily transferred to the personal computer (PC) over the USB-link for data-analysis.

Owing to the flexible and lightweight design of the system, the PDE is comfortably wearable by the test persons without restricting their movements. A top-view of the exposimeter circuit is shown in Fig. 4. Its planar circuit board's size of

TABLE I

SPECIFICATIONS OF THE SINGLE PEM NODE

\begin{tabular}{ll}
\hline \hline Specification & Value \\
\hline Dynamic Range & $80 \mathrm{~dB}$ \\
Minimum Input Power & $-70 \mathrm{dBm}$ \\
Maximum Input Power & $10 \mathrm{dBm}$ \\
Operating supply voltage & $3.5-12 \mathrm{~V}$ \\
Size antenna & $100 \mathrm{~mm} \times 125 \mathrm{~mm}$ \\
Size PCB & $35 \mathrm{~mm} \times 55 \mathrm{~mm}$ \\
Frequency range & $1 \mathrm{MHz}-4 \mathrm{GHz}$ \\
\hline
\end{tabular}

$35 \mathrm{~mm}$ by $55 \mathrm{~mm}$ is smaller than the antenna, allowing easy integration onto its feed-plane. An overview of the technical specifications is shown in Table I.

\section{Frequency selection of the personal exposimeter}

The measured frequency band is selected by the bandpass Surface Acoustic Wave (SAW) filter. For each desired frequency band, a filter with the appropriate response may be inserted, without needing to adjust the circuit design. In this application, the full GSM 900 frequency band is measured, without further adjustments to the circuit or antenna, allowing to measure the incident power density in this particular frequency band.

\section{Calibration}

The digitized output voltage of the logarithmic-detector is a function of the corresponding RF input power. This function is accurately determined by means of calibration.

The calibration of the RF input level of the exposimeter without antenna is performed in an anechoic chamber. Calibration datasets are constructed for each unit separately and stored into its flash memory for use during the actual exposure measurements. Logarithmic detection results in an accurate measurement over a large dynamic range, stored in a limited number of bits per measurement value. The Logarithmic RF Detector exhibits $80 \mathrm{~dB}$ dynamic range, with a minimum $\mathrm{RF}$ input level of $-70 \mathrm{dBm}$. By employing the calibration data, a $1 \mathrm{~dB}$ resolution is achieved. To compensate the temperature-dependent offset, the output voltage of the DAC (Analog Devices AD5641 [22]) is automatically adjusted [19] as a function of the operating temperature.

\section{E. Exposimeter synchronization}

In the proposed PDE setup, where more than one exposimeter node is employed on the human test person, synchronization of all the exposimeter nodes is required to achieve an exposure measurement with accurate timestamps. All the exposimeter nodes are equipped with the same 24.576 MHz crystal with a frequency stability of $10 \mathrm{PPM}$. The sample period is directly derived from this main on-board clock, thereby minimizing the influence of frequency instability over a long time. Synchronization is achieved by connecting each individual exposimeter node to the PC. The $\mathrm{PC}$ will initialize the timing registers of the microcontroller for deriving the sample period. In addition, a time stamp is placed into the flash memory based on the PC clock. This ensures that all modules composing the exposimeter will sample at the same time instant within the defined sample period, with only a minimal deviation. After synchronization, each individual exposimeter immediately starts capturing exposure data. When the measurement campaign is terminated by the user, the data are transferred to the $\mathrm{PC}$, including the time stamps in the

TABLE II

CURRENT CONSUMPTION OF THE MAIN COMPONENTS OF THE EXPOSIMETER NODE

\begin{tabular}{llll}
\hline \hline \multirow{2}{*}{ Component } & PowER MODE & $\begin{array}{l}\text { CURRENT } \\
\text { CONSUMPTION }\end{array}$ & $\begin{array}{l}\text { POWER } \\
\text { @ 3V3 }\end{array}$ \\
\hline \multirow{3}{*}{ Microcontroller } & Idle & $2.5 \mathrm{~mA}$ & $8.25 \mathrm{~mW}$ \\
& Sleep & $0.1 \mu \mathrm{A}$ & $0.33 \mu \mathrm{W}$ \\
& Normal & $4 \mathrm{~mA}$ & $13.2 \mathrm{~mW}$ \\
\hline \multirow{2}{*}{ RF detector } & Power on & $31 \mathrm{~mA}$ & $102.3 \mathrm{~mW}$ \\
& Power down & $<200 \mu \mathrm{A}$ & $<660 \mu \mathrm{W}$ \\
\hline \multirow{2}{*}{ Memory } & Stand by & $25 \mu \mathrm{A}$ & $82.5 \mu \mathrm{W}$ \\
& Deep Power-down & $5 \mu \mathrm{A}$ & $16.5 \mu \mathrm{W}$ \\
& Read/Write & $12 \mathrm{~mA}$ & $39.6 \mathrm{~mW}$ \\
\hline \multirow{2}{*}{ ADC } & Power on at 100 ksps & $550 \mu \mathrm{A}$ & $1.82 \mathrm{~mW}$ \\
& Stand by & $1 \mathrm{nA}$ & $3.3 \mathrm{nW}$ \\
\hline \multirow{2}{*}{ Temp comp. } & Normal Mode & $60 \mu \mathrm{A}$ & $198 \mu \mathrm{W}$ \\
& All Power down & $500 \mathrm{nA}$ & $1.65 \mu \mathrm{W}$ \\
\hline \hline
\end{tabular}

flash memory. Based on the timestamps, the processing software aligns the data samples and starts further data processing.

\section{F. Power consumption}

The exposimeter is powered by a 1-cell Lithium polymer (Li-po) battery and a low-drop linear voltage regulator. From the technical data sheets of the integrated components, an estimation of the power consumption is made. An overview of that current consumption is given in Table II.

The microcontroller will consume an average current of 4 $\mathrm{mA}$ at $3.3 \mathrm{~V}$ and at a clock frequency $24.576 \mathrm{MHz}$. In sleep mode, the current consumption can be reduced to $600 \mathrm{nA}$. The flash memory consumes on average $12 \mathrm{~mA}$ at $3.3 \mathrm{~V}$ while operating (reading or writing), whereas in standby mode, the current consumption is reduced to $25 \mu \mathrm{A}$, or to $5 \mu \mathrm{A}$ in deep 
power-down mode.

The most current-consuming device on the exposimeter node is the RF detector, consuming $31 \mathrm{~mA}$ in full operation. In power down mode, its current consumption is lowered to less than $200 \mu \mathrm{A}$. The current consumption of the ADC and temperature compensation circuits is $550 \mu \mathrm{A}$ and $60 \mu \mathrm{A}$, respectively.

The average current consumption is estimated to be $40 \mathrm{~mA}$ at $3.3 \mathrm{~V}$, taking into account that the detector is always in power on mode, while the memory is accessed only once every second.

In full operation, the measured average power consumption of one exposimeter node equals $131 \mathrm{~mW}$ (39.6 mA current consumption, at $3.3 \mathrm{~V}$ supply voltage). This enables the sensor network to operate for many hours, without the need for charging the battery. The power consumption can further be reduced by employing the sleep mode of the system when there is no need to continuously operate at high speed.

\section{VALIDATION}

\section{A. Free-space performance}

To measure the frequency response at the input of the exposimeter, the complete sensor (including vertically polarized antenna) is placed in an anechoic chamber at $4.34 \mathrm{~m}$ from the radiating antenna. The TX standard gain horn (NSIRFSG975, with a gain of $14 \mathrm{dBi}$ at $942.5 \mathrm{MHz}$ ), connected to a signal source with an output power of $10 \mathrm{dBm}$ (cable losses $=3.75 \mathrm{~dB}$ ), swept over a frequency range from $840 \mathrm{MHz}$ to $1040 \mathrm{MHz}$, is radiating along both horizontal and vertical TX polarizations. The response is shown in Fig. 5, indicating a large attenuation for out-of-band signals. Clearly, the attenuation is very steep on the bottom side of the GSM downlink band, resulting in a rejection by at least $35 \mathrm{~dB}$ of 880-915 MHz GSM-900 uplink signals. For frequencies slightly above the GSM-900 downlink band, a better than $23 \mathrm{~dB}$ attenuation is also sufficient, considering that, according to the band planning, no strong signals are expected adjacent to the upper end of the GSM downlink band. Since the measured received power on the exposimeter in this anechoic measurement is significantly larger than the signals that will actually be measured during a real-world measurement, out-of-band signals will be below the noise floor of the exposimeter thanks to the band-pass filtering characteristics. They will not affect the actual measurements in the desired frequency band.

The clearly visible difference of approximately $12 \mathrm{~dB}$ between both TX polarizations is due to the vertically polarized receive antenna of the node. An ideal exposimeter has no dependence of the polarization. In order to minimize the influence of the received polarizations, several exposimeters are placed onto the body, oriented along orthogonal polarizations, as further described and evaluated.

While the transmitted signal of the base station is vertically polarized, the received polarization will vary due to the angle of arrival on the nodes and the different paths followed by the signal in the environment. By orienting the nodes along both polarizations, all signals received from the base station can be captured. Furthermore, these linearly polarized antennas are

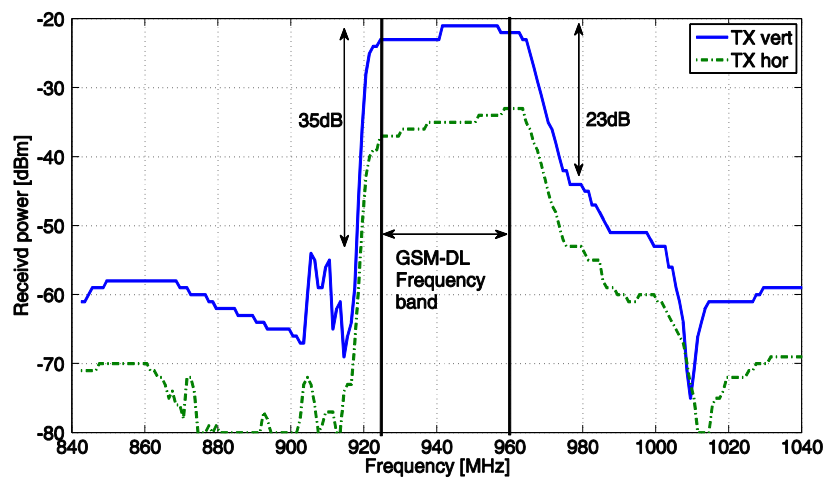

Fig. 5. Free-space frequency response of the vertically polarized exposimeter

easier to construct, in comparison to textile patch antennas

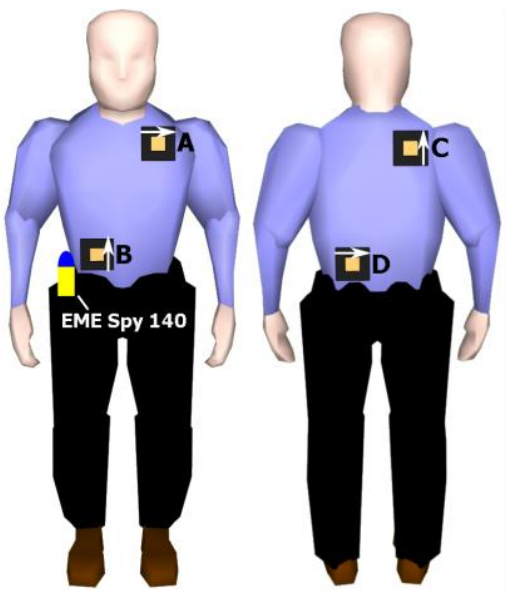

Fig. 6. Positions of the four Personal ExposiMeter (PEM) modules composing the Personal Distributed Exposimeter (PDE), shown together with the position of the EME Spy 140 onto the body of the test-person

with a circular polarization, thereby reducing the cost and the size of the nodes.

\section{B. On-body performance}

The PDE is configured for the GSM-900 down link frequency band, which is present in most environments. Four separate nodes of the PDE are distributed at optimal positions over the front and rear sections of the torso of a $1.85 \mathrm{~m}$ large test person having a weight of $80 \mathrm{~kg}$, as shown in Fig. 6. The polarizations of the individual nodes are also chosen for complementarity.

The person with the four nodes distributed over the body stands on the rotor inside the anechoic chamber, in the farfield of the standard gain horn, radiating at $942.5 \mathrm{MHz}$, being the center frequency of the GSM-900 down-link band, connected to a signal generator with a transmit power of $10 \mathrm{dBm}$ (cable losses $=3.75 \mathrm{~dB}$ ). The person wearing the PDE is rotated in the azimuth plane over an azimuth angle of $360^{\circ}$. These measurements are repeated for Horizontal $(\mathrm{H})$ and Vertical (V) TX polarizations. During these measurements, a commercially available secondary exposimeter 


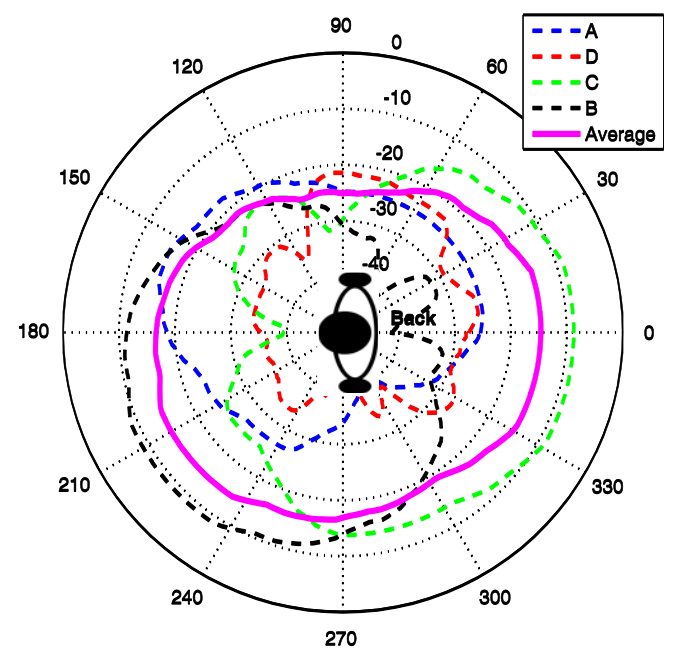

Fig. 7. Electric field strength $[\mathrm{dB}]$ on all 4 nodes in the azimuth plane, worn on-body as shown in Fig. $6.180^{\circ}=$ front side of the body, vertically polarized TX antenna

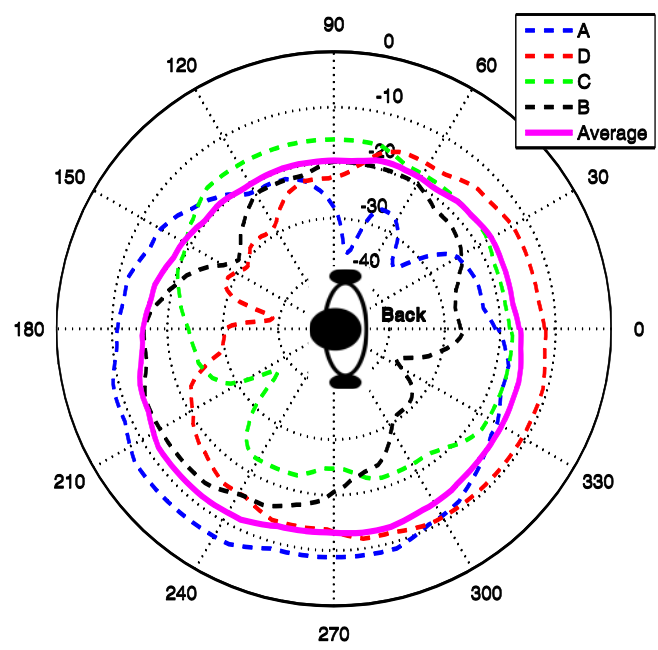

Fig. 8. Electric field strength $[\mathrm{dB}]$ on all 4 nodes in the azimuth plane, worn on-body as shown in Fig. $6.180^{\circ}=$ front side of the body, horizontally polarized TX antenna

(EME Spy 140) is worn at waist-height. The distance from the middle of the human test subject to the aperture of the horn antenna is $4.34 \mathrm{~m}$.

These measurements are plotted in a logarithmic scale, shown in Figs. 7 and 8, for TX Vertical and Horizontal polarizations, respectively. Besides the field strength on each single node, the averaged field strength over the four nodes is calculated in the azimuth plane for each azimuth angle $\varphi$, for both horizontal and vertical polarizations. The average field strength of the proposed PDE, calculated for each angle in the azimuth plane, is approximately constant, making the exposimeter output independent of the transmit polarization.

In order to determine the dependence on the polarization when worn on-body, the standard deviation $\sigma_{\mathrm{pol}}$ is calculated based on the difference in the received field strength of both polarizations. It is determined for both the "EME Spy 140" and for the PDE, by averaging the logarithmic field strength over the four nodes. This results in

$$
\begin{aligned}
\sigma_{P D E \text { diff } H / V} & =2.32 \mathrm{~dB} \\
\sigma_{\text {EME Spyl 40diff } H / V} & =3.73 \mathrm{~dB}
\end{aligned}
$$

This allows us to conclude that the PDE is less polarization dependent than the "EME Spy 140". To compare the PDE and the "EME Spy 140" in an on-body scenario, the standard deviation $\sigma$ of the field strength over different azimuth angles is calculated for both horizontal as well as vertical polarization. $\sigma$ indicates how circular the pattern is in azimuth angle. A pattern that is perfectly omnidirectional results in a standard deviation of $0 \mathrm{~dB} . \sigma_{\varphi}$ is derived from the (Logarithmic) field strength on the four nodes, resulting in

$$
\begin{aligned}
\sigma_{P D E \text { Hor }} & =2.70 \mathrm{~dB} \\
\sigma_{P D E \text { Vert }} & =3.35 \mathrm{~dB} \\
\sigma_{\text {EME Spy140Hor }} & =6.94 \mathrm{~dB} \\
\sigma_{\text {EME Spy140Vert }} & =9.13 \mathrm{~dB}
\end{aligned}
$$

We clearly obtain a better performance for the PDE in comparison to the commercial exposimeter. In Figs. 9 and 10, the normalized field strengths, for both the PDE and the "EME Spy 140", are plotted for a vertically and horizontally

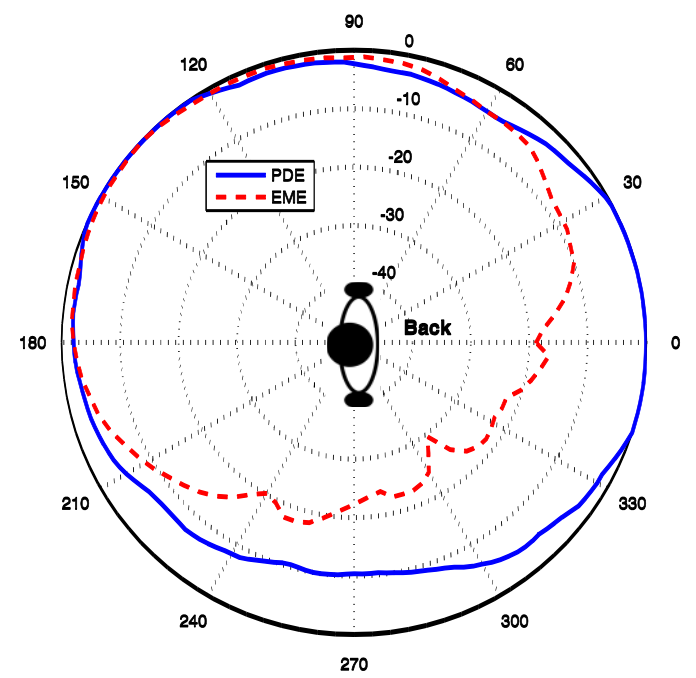

Fig. 9. Normalized electric field strength $[\mathrm{dB}]$ on the PDE and "EME Spy $140^{\prime \prime} .180^{\circ}=$ front side of the body, TX vertical polarization

polarized transmitted signal, respectively. This visually verifies the above results. The PDE clearly achieves a more uniform distribution of the field strength over the azimuth plane.

\section{CAlibration}

As discussed above, the power received by the PDE is almost constant, independent of polarization or azimuth angle for a given transmit power. To obtain an accurate measurement result of the actual RF field strength at the location of the human body in a real environment, the PDE requires calibration, which is performed in an anechoic chamber. The calibration eliminates the influence of the body on the PDE measurement.

The measurements in the anechoic chamber, for both horizontal and vertical TX polarizations, used earlier to 


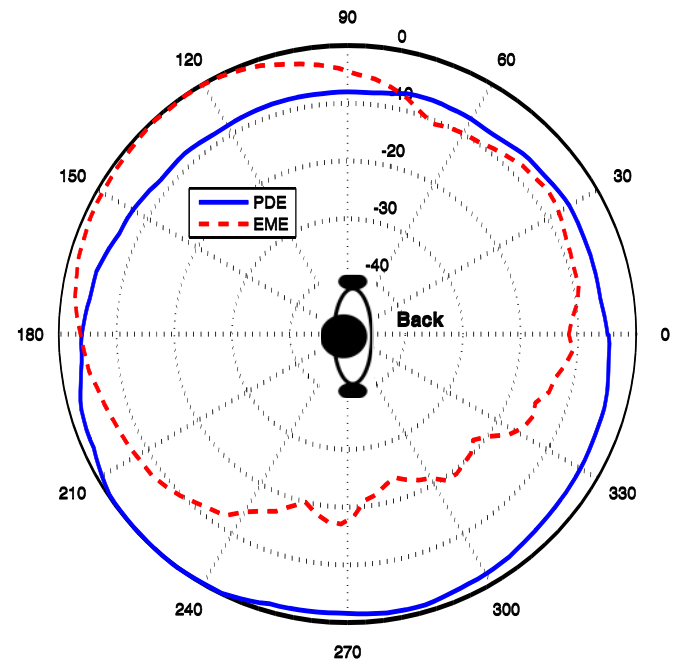

Fig. 10. Normalized electric field strength $[\mathrm{dB}]$ on the PDE and "EME Spy $140 " .180^{\circ}=$ front side of the body, TX horizontal polarization

validate the exposimeters, are now employed to calibrate the PDE when performing on-body measurements. $\mathrm{P}_{\text {geom }}^{\mathrm{H}}(\varphi)$ and $\mathrm{P}^{\mathrm{V}}{ }_{\text {geom }}(\varphi)$ are the geometric average received powers for the horizontally and vertically transmit polarization as a function of the azimuth angle, respectively. Furthermore, the free-space incident powers $\mathrm{S}^{\mathrm{H}}$ inc and $\mathrm{S}_{\text {inc }}^{\mathrm{V}}$ are measured using the NBM550 broadband probe, for both polarizations at the TX horn antenna, as described in section III.B for the PDE, but now with the broadband probe at exactly the same location.

From the calibration measurements, the geometric average Antenna Aperture $\left(\mathrm{AA}_{\text {geom }}\right)$ of the total PDE is determined, given by

$$
A A_{\text {geom }}(\varphi, \psi)=\frac{P_{\text {geom }}^{H}(\varphi)}{S_{\text {inc }}^{H}} \cos ^{2}(\psi)+\frac{P_{\text {geom }}^{V}(\varphi)}{S_{\text {inc }}^{V}} \sin ^{2}(\psi)
$$

where $\mathrm{S}_{\text {inc }}^{\mathrm{V}}=0.541 \mathrm{~mW} / \mathrm{m}^{2}$ and $\mathrm{S}_{\text {inc }}^{\mathrm{H}}=0.394 \mathrm{~mW} / \mathrm{m}^{2}$, and with $\psi$ the polarization of an incident electric field. $\mathrm{AA}_{\text {geom }}(\varphi$, $\psi)$ is calculated for $10^{3} \varphi$-samples, located in the interval [0-
$2 \pi$ ] radians. The $\psi$ samples are drawn from a Gaussian distribution in an "Urban Macro cell" scenario [3], [23], in order to take into account a realistic polarization of the incident electric field. This scenario provides the best correspondence to earlier measurements performed in the city of Ghent [6].

The set of values resulting from this procedure provides the distribution of $\mathrm{AA}_{\text {geom }}$ for realistic angles of arrival. From this distribution, the median is chosen as the value of the Antenna Aperture $\left(\mathrm{AA}_{\text {geom }}\right)$ of the total PDE. In addition, the full calibration procedure is repeated 100 times, and the results are averaged in order to improve accuracy. Based on this calibration process, the average value of $\mathrm{AA}_{\text {geom }}$ is found to be $6.58 \mathrm{~cm}^{2}$.

Once the value of $\mathrm{AA}_{\text {geom }}$ is determined, a real world measurement can start. After this measurement, the incident power received on the body of the test person can be determined by

$$
S_{\text {inc }}=\frac{P_{\text {geom }}}{A A_{\text {geom }}}
$$

where $\mathrm{P}_{\text {geom }}$ is the geometric average received power on the four nodes of the PDE, observed during the real world measurement.

\section{REAL-WORLD MEASUREMENTS}

To perform a real-world measurement, the same test person as in previous measurements, equipped with the PDE and the "EME Spy 140", walks along a predefined path in the citycenter of Ghent (Belgium). During this walk, the received power is recorded on all the exposimeter nodes as well as on the "EME Spy 140", in a time interval of 1 second. Fig. 11 shows the total power density received by the test person with the calibrated PDE during the complete walk, as well as the power density on the "EME Spy 140 ". In Fig. 12, the $2.6 \mathrm{~km}$ outdoor trajectory through Ghent, followed by the test person, is shown. On this map, the position markers corresponding to

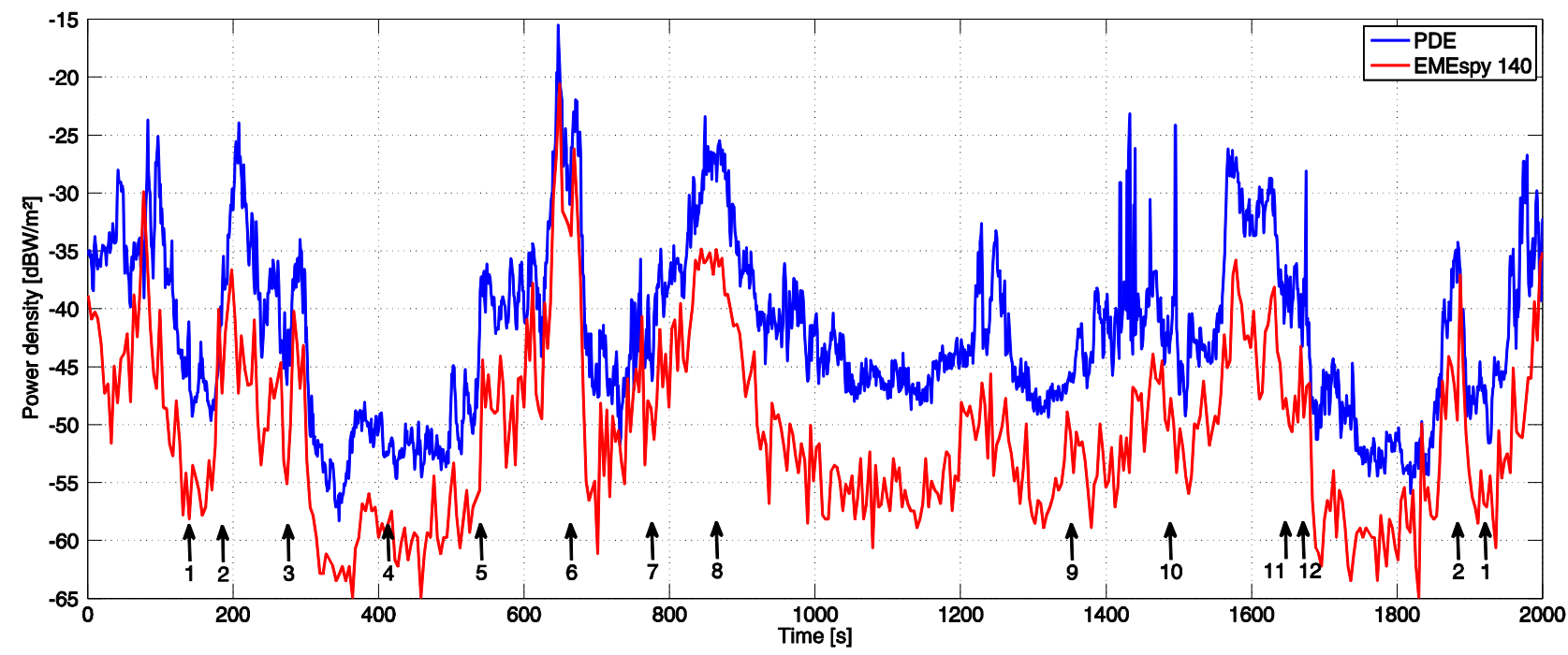

Fig. 11. Received power density $\left[\mathrm{dBW} / \mathrm{m}^{2}\right]$ on the body of the test person, measured with the active nodes of the PDE versus the commercially available exposimeter. The position markers corresponding to the numbers in Fig. 12. 
the numbers in Fig. 11, as well as the position of the nearby GSM-900 base-stations [24], are shown.

The total power density, received during the walk, is determined based on the active exposimeter nodes of the PDE, after applying the calibration procedure described in Section IV. The power density of the "EME Spy 140" is extracted from the measurement logging file. The measurement results clearly show that the received powers of both measurement devices exhibit the same trend, but with short-term differences in power density levels. The shadowing by the body has a significant influence on the measurement results by the commercial "EME Spy 140". As stated earlier in Section III, the signals received on the "EME Spy 140" are dependent on the angle of arrival of the signals in the azimuth plane. The omnidirectional receive pattern, which is obtained with the PDE, ensures a more accurate estimation of the power density levels in comparison to a non-distributed device such as the "EME Spy 140". To verify whether the "EME Spy 140" indeed underestimates the RF field exposure levels, two additional experiments were performed. To demonstrate that the underestimation due to body shadowing occurs in a general case, the experiments were performed in a different part of the city. First, the "EME Spy 140" was deployed in two setups. On the one hand, an on-body measurement was performed by wearing the exposimeter near the waist of the user. On the other hand, the exposimeter was held above the

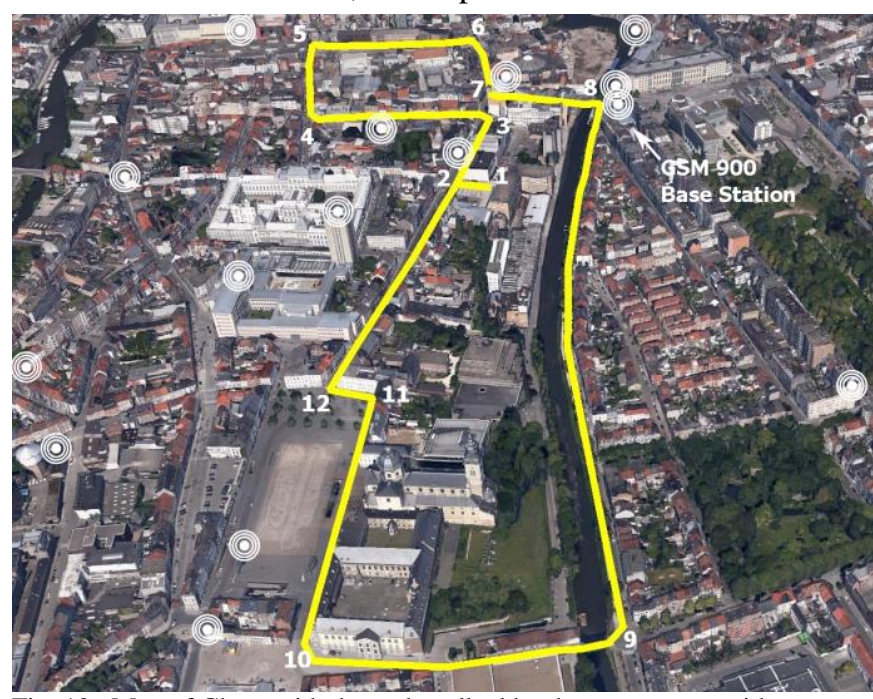

Fig. 12. Map of Ghent with the path walked by the test person, with numbered position markers and GSM-900 basestation symbols, (source: Google Earth)

head, carrying out a measurement that is certainly is less influenced by the body. During the on-body measurement, an average power density level of $-45.5 \mathrm{dBW} / \mathrm{m}^{2}$ was measured. In contrast, the second measurement yielded an average power density level of $-43 \mathrm{dBW} / \mathrm{m}^{2}$. Hence, a difference of $2.5 \mathrm{~dB}$ was obtained between both situations, clearly showing the influence of the body on the exposimeter, resulting in an underestimation of the exposure. Next, static measurements were performed by the "EME Spy 140" on a tripod, by a spectrum analyzer with a calibrated reference antenna (Rohde \& Schwarz TSEMF-B1), and by our PDE, worn onbody. All devices were positioned in the line of sight of a
GSM base station The spectrum analyser measured an average power density of $-37.4 \mathrm{dBW} / \mathrm{m}^{2}$, whereas the "EME Spy 140 " yielded an average power density of $-41.9 \mathrm{dBW} / \mathrm{m}^{2}$. The $4.5 \mathrm{~dB}$ difference in the results suggests that the EME slightly underestimates the field strengths, even without the presence of the human body. We conclude that the total difference of $7.0 \mathrm{~dB}$, between the spectrum analyser and the "EME Spy 140" on the body, approximately corresponds to the difference between the PDE and the on-body "EME Spy 140". The average power density level measured by the on-body PDE equals $-38.0 \mathrm{dBW} / \mathrm{m}^{2}$, yielding only a slight measurement difference of $0.6 \mathrm{~dB}$ with respect to the spectrum analyser. Table III summarizes these average power density levels, obtained during the static measurements campaign.

TABLE III

AVERAGE POWER DENSITY LEVELS OBTAINED DURING STATIC MEASUREMENT

\begin{tabular}{ll}
\hline \hline Measurement device & Value \\
\hline Spectrum analyser & $-37.4 \mathrm{dBW} / \mathrm{m}^{2}$ \\
Personal Distributed Exposimeter & $-38.0 \mathrm{dBW} / \mathrm{m}^{2}$ \\
EME Spy 140 & $-41.9 \mathrm{dBW} / \mathrm{m}^{2}$ \\
\hline \hline
\end{tabular}

The maximum instantaneous power density measured by the PDE during the experiment is $28 \mathrm{~mW} / \mathrm{m}^{2}$. As a result, the average power density levels over a 6-minute time frame are well below the ICNIRP reference level of $4.8 \mathrm{~W} / \mathrm{m}^{2}$ [1]. Furthermore, from these measurements, the $\mathrm{SAR}_{\mathrm{wb}}$ can be determined, as described in [17],[18].

\section{CONCLUSION}

A compact wearable Personal Distributed Exposimeter is proposed, which increases the measurement accuracy in comparison to conventional Personal Exposimeters, including the dependency on the polarization and the angle in the azimuth plane. The Personal Distributed Exposimeter is composed of several newly designed on-body exposimeter modules, which are integrated onto the feed plane of a textile antenna. The different modules apply synchronous exposure data sampling, while being unobtrusively integrated inside a garment and being distributed over the body of the test person. Therefore, this new compact exposimeter is a step forward towards user-friendly Personal Distributed Exposimeters in multiple frequency bands, integrated into a single garment for measuring exposure data in a convenient way. Validation of the Personal Distributed Exposimeter shows that the system exhibits less dependence of the received polarization or the angle of the azimuth plane, compared to commercial available exposimeters. A fast and accurate calibration process is proposed, to eliminate the influence of the body onto the PDE.

To validate the measurements performed by the Personal Distributed Exposimeter, a real world exposure measurement was carried out for the GSM-900 downlink band. The measurement is performed in the city center of Ghent, whose propagation characteristics correspond to an Urban Macro Cell. The measurement was also carried out employing an "EME Spy 140" commercial exposimeter. This experiment clearly illustrates that the PDE provides a more accurate 
estimation of the power density levels on the human body. The commercial, non-calibrated exposimeter deployed on the body influences the measurement results due to shadowing by proximity of the body, leading to an underestimation of the power density levels on the human body. The maximum instantaneous power density measured by the PDE during the experiment equals $28 \mathrm{~mW} / \mathrm{m}^{2}$, which is well below the ICNIRP reference level of $4.8 \mathrm{~W} / \mathrm{m}^{2}$ for an average power density level in a 6-minute time frame. Based on these measurement data, the whole-body SAR is readily determined.

Besides for verifying compliance of RF field exposure with ICNIRP reference levels, the proposed modules can also serve as sensor nodes to evaluate the potential of RF energy harvesting [25]-[28] and wireless power transfer [29].

\section{REFERENCES}

[1] International Commission on Non-Ionizing Radiation Protection, "Guidelines for limiting exposure to time-varying electric, magnetic, and eletromagnetic fields (up to $300 \mathrm{GHz}$ )", Health Physics, vol. 74, pp. 494-552, 1998 .

[2] Wout Joseph, Günter Vermeeren, Leen Verloock, and Luc Martens, "Estimation of whole-body SAR from electromagnetic fields using personal exposure meters", Bioelectromagnetics, vol. 31, no. 4, pp. 286295, 2010.

[3] Günter Vermeeren, Wout Joseph, Christof Olivier, and Luc Martens, "Statistical multipath exposure of a human in a realistic electromagnetic environment", Health Physics, vol. 94, no. 4, pp. 345-354, 2008.

[4] Damiano Urbinello, Anke Huss, Johan Beekhuizen, Roel Vermeulen, and Martin Röösli, "Use of portable exposure meters for comparing mobile phone base station radiation in different types of areas in the cities of Basel and Amsterdam", Science of The Total Environment, vol. 468469, pp. 1028 - 1033, 2014.

[5] György Thuróczy, Ferenc Molnár, Gábor Jánossy, Noémi Nagy, Györgyi Kubinyi, József Bakos, and Judit Szabó , "Personal RF exposimetry in urban area", Annals of telecommunications - Annales des télécommunications, vol. 63, no. 1-2, pp. 87-96, 2008.

[6] Wout Joseph, Günter Vermeeren, Leen Verloock, Mauricio Masache Heredia, and Luc Martens, "Characterization of personal RF electromagnetic field exposure and actual absorption for the general public", Health Physics, vol. 95, no. 3, pp. 317-330, 2008.

[7] Arno Thielens, Sam Agneessens, Leen Verloock, Emmeric Tanghe, Hendrik Rogier, Luc Martens, and Wout Joseph, "On-body calibration and processing for a combination of two radio-frequency personal exposimeters", Radiation Protection Dosimetry, in press.

[8] S. Iskra, R. McKenzie, and I. Cosic, "Factors influencing uncertainty in measurement of electric fields close to the body in personal RF dosimetry", Radiation Protection Dosimetry, 2010.

[9] John F.B. Bolte, Gerard van der Zande, and Jos Kamer, "Calibration and uncertainties in personal exposure measurements of radiofrequency electromagnetic fields", Bioelectromagnetics, vol. 32, no. 8, pp. 652$663,2011$.

[10] John F.B. Bolte and Tessa Eikelboom, "Personal radiofrequency electromagnetic field measurements in the Netherlands: Exposure level and variability for everyday activities, times of day and types of area", Environment International, vol. 48, pp. 133 - 142, 2012.

[11] Arno Thielens, Hans De Clercq, Sam Agneessens, Jeroen Lecoutere, Leen Verloock, Frederick Declercq, Günter Vermeeren, Emmeric Tanghe, Hendrik Rogier, Robert Puers, Luc Martens, and Wout Joseph, "Personal distributed exposimeter for radio frequency exposure assessment in real environments", Bioelectromagnetics, vol. 34, no. 7, pp. 563-567, 2013.

[12] P. Salonen, L. Sydanheimo, M. Keskilammi, and M. Kivikoski, "A small planar inverted-F antenna for wearable applications", in Wearable Computers, 1999. Digest of Papers. The Third International Symposium on, Oct 1999, pp. 95-100.

[13] Wout Joseph, Günter Vermeeren, Leen Verloock, and Luc Martens, "Estimation of whole-body SAR from electromagnetic fields using personal exposure meters", Bioelectromagnetics, vol. 31, no. 4, pp. 286$295,2010$.
[14] Frederick Declercq, A. Georgiadis, and Hendrik Rogier, "Wearable aperture-coupled shorted solar patch antenna for remote tracking and monitoring applications", in Proceedings of the 5th European conference on Antennas and propagation EUCAP. 2011, pp. 29922996, IEEE.

[15] C. Hertleer, A. Tronquo, H. Rogier, L. Vallozzi, and L. Van Langenhove, "Aperture-coupled patch antenna for integration into wearable textile systems", Antennas and Wireless Propagation Letters, IEEE, vol. 6, pp. 392-395, 2007.

[16] V. Rathi, G. Kumar, and K. P. Ray, "Improved coupling for aperture coupled microstrip antennas", Antennas and Propagation, IEEE Transactions on, vol. 44, no. 8, pp. 1196-1198, Aug 1996.

[17] Arno Thielens, Peter Vanveerdeghem, Sam Agneessens, Patrick Van Torre, Günter Vermeeren, Hendrik Rogier, Luc Martens, and Wout Joseph, "Whole-body averaged SAR assessment using a personal, distributed exposimeter", in Proceedings of BioEM2014. 2014, p. 6, The Bioelectromagnetics Society (BEMS).

[18] Arno Thielens, Peter Vanveerdeghem, Sam Agneessens, Patrick Van Torre, Günter Vermeeren, Hendrik Rogier, Luc Martens, and Wout Joseph, "Whole-Body Averaged Specific Absorption Rate Estimation using a Personal, Distributed Exposimeter", Accepted for publication in Antennas and Wireless Propagation Letters, IEEE, 2014.

[19] Peter Vanveerdeghem, Patrick Van Torre, Christiaan Stevens, Jos Knockaert, and Hendrik Rogier, "Flexible dual-diversity wearable wireless node integrated on a dual-polarised textile patch antenna", IET Science, Measurement \& Technology, May 2014.

[20] M.L. Scarpello, I. Kazani, C. Hertleer, H. Rogier, and D. Vande Ginste, "Stability and Efficiency of Screen-Printed Wearable and Washable Antennas", Antennas and Wireless Propagation Letters, IEEE, vol. 11, pp. 838-841, 2012.

[21] Analog Devices, "ADL5513 datasheet rev 0", Tech. Rep., Analog Devices, One Technology Way, P.O. Box 9106, Norwood, MA 020629106, U.S.A., 2008.

[22] Analog Devices, "AD5641 datasheet rev d", Tech. Rep., Analog Devices, One Technology Way, P.O. Box 9106, Norwood, MA 02062-9106, U.S.A., 2008.

[23] Arno Thielens, Günter Vermeeren, Wout Joseph, and Luc Martens, "Stochastic method for determination of the organ-specific averaged SAR in realistic environments at $950 \mathrm{MHz}$ ", Bioelectromagnetics, vol. 34, no. 7, pp. 549-562, 2013.

[24] Belgian Institute for Postal services and Telecommunications, "Antenna site register", 2014.

[25] T. Le, K. Mayaram, and T. Fiez, "Efficient far-field radio frequency energy harvesting for passively powered sensor networks", Solid-State Circuits, IEEE Journal of, vol. 43, no. 5, pp. 1287-1302, May 2008.

[26] J. Zbitou, M. Latrach, and Serge Toutain, "Hybrid rectenna and monolithic integrated zero-bias microwave rectifier", Microwave Theory and Techniques, IEEE Transactions on, vol. 54, no. 1, pp. 147-152, Jan 2006.

[27] G. Andia Vera, A. Georgiadis, A. Collado, and S. Via, "Design of a 2.45 $\mathrm{GHz}$ rectenna for electromagnetic (EM) energy scavenging", in Radio and Wireless Symposium (RWS), 2010 IEEE, Jan 2010, pp. 61-64.

[28] Antwi Nimo, Dario Grgi, and Leonhard M. Reindl, "Optimization of passive low power wireless electromagnetic energy harvesters", Sensors, vol. 12, no. 10, pp. 13636-13663, 2012.

[29] N. Carvalho, A. Georgiadis, A. Costanzo, H. Rogier, A. Collado, J. A. García, S. Lucyszyn, P. Mezzanotte, J. Kracek, D. Masotti, A. Boaventura, M. Nieves Ruíz, M. Pinuela, D. Yates, P. Mitcheson, M. Mazanek, and V. Pankrac, "Wireless Power Transmission: R\&D Activities within Europe", IEEE Trans. Microwave Theory Tech., vol. 62, no. 4, pp. 1031-1045, Apr. 2014. 


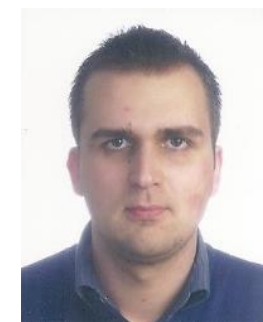

Peter Vanveerdeghem was born in 1986. He received the $M . S c$. degree in Electronics Engineering from the University College West-Flanders, Belgium, in 2008, the M. Sc. degree in Electrical Engineering and the Ph.D. degree from Ghent University, Ghent, Belgium, in 2011 and 2015 respectively. His research interests are the design of embedded systems and transceivers on textile-antenna platforms as well as body-centric wireless communication.

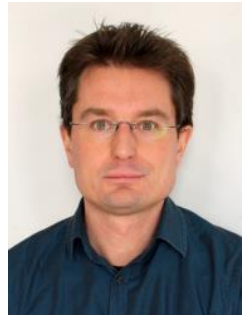

Patrick Van Torre received the Electrical Engineering degree (ing.) in 1995 and obtained the Ph.D. degree at Ghent University, Gent, Belgium in 2012. He is employed as a lecturer and postdoctoral researcher with the Department of Information Technology at Ghent University. He lectures theory courses in Electronics and ICT, organizes project-oriented lab sessions and is involved in public relations activities as well as hardware development projects for third parties. As a researcher, he is active in the field of wireless communication, focusing on body-centric multiple-input multiple-output (MIMO) and beam-forming systems, the design of radiofrequency exposimeters as well as the integration of embedded systems and transceivers on textile-antenna platforms.

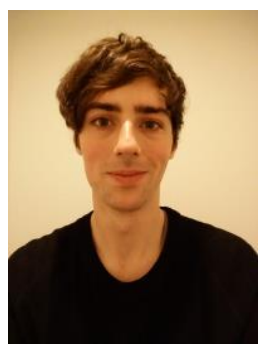

Arno Thielens was born in Brasschaat, Belgium, on November 12, 1987. He received the $M$. Sc. degree in applied physics from Ghent University, Ghent, Belgium, in 2010. Since October 2010, he has been a Research Assistant with iMinds/UGent-INTEC

(Ghent

University-Department of Information Technology). His scientific work focusses on numerical dosimetry and exposure assessment of radio frequency electromagnetic fields.

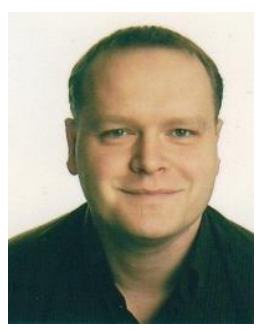

Jos Knockaert received the degree of industrial engineer in Electrotechnics in 1996 from the University College of Bruges-Ostend, the M.Sc. degree in Electronic System Design in 2001 from Leeds Metropolitan University and the Ph.D. degree in Engineering Science in 2009 from the K.U. Leuven. He worked as EMC-design engineer in the industry and became assistant at the University College of BrugesOstend. Since 2010, he is assistant professor at Ghent University, teaching electrical machines, electromagnetic compatibility and power electronics. He is member of the research group Lemcko with focus on electromagnetic compatibility, power electronics and high frequency problems in industrial systems.

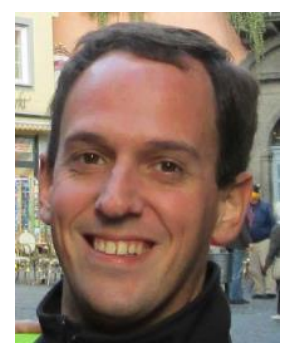

Wout Joseph (M'05-SM'12) was born in Ostend, Belgium, on October 21, 1977. He received the M. Sc. degree in electrical engineering and the Ph.D. degree from Ghent University (UGent), Ghent, Belgium, in 2000 and 2005, respectively. From September 2000 to March 2005, he was a Research Assistant with the iMinds/UGentINTEC (Ghent University - Department of Information Technology). During this period, his scientific work was focused on electromagnetic exposure assessment. His research work dealt with measuring and modeling of electromagnetic fields around base stations for mobile communications related to the health effects of the exposure to electromagnetic radiation. This work led to the Ph.D. degree. Since April 2005, he has been a Postdoctoral Researcher with iMindsUGent/INTEC. From October 2007 to October 2013, he was a Postdoctoral Fellow of Research Foundation- Flanders (FWOV). Since October 2009, he has been a Professor in the domain of experimental characterization of wireless communication systems. His professional interests are electromagnetic field exposure assessment, propagation for wireless communication systems, antennas, and calibration. Furthermore, he specializes in wireless performance analysis and quality of experience.

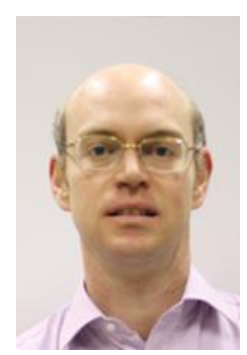

Hendrik Rogier was born in 1971. He received the Electrical Engineering and the Ph.D. degrees from Ghent University, Gent, Belgium, in 1994 and in 1999, respectively. $\mathrm{He}$ is a currently a Full Professor with the Department of Information Technology of Ghent University, Belgium, Guest Professor at IMEC, Heverlee, Belgium, and Visiting Professor at the University of Buckingham, UK. From October 2003 to April 2004, he was a Visiting Scientist at the Mobile Communications Group of Vienna University of Technology. He authored and coauthored about 90 papers in international journals and about 110 contributions in conference proceedings. He is serving as a member of the Editorial Board of IET Science, Measurement Technology and acts as the URSI Commission B representative for Belgium. Within the IEEE Microwave Theory and Techniques Society, he is a member of Technical Committee 24 on RFID technology and within the European Microwave Association, he is a member of the Governing Board of Topical Group MAGEO on Microwaves in Agriculture, Environment and Earth Observation. His current research interests are antenna systems, radiowave propagation, body-centric communication, numerical electromagnetics, electromagnetic compatibility and power/signal integrity. 